\title{
Regulated bioanalysis and the desire for harmonized regulations in China
}

\section{"...there is a great desire for the bioanalytical laboratories in China to follow the same harmonized bioanalytical regulation if possible."}

Keywords: bioanalysis $\approx$ bioanalytical method validation guidelines $₫$ global $₫$ harmonization $\approx$ regulations

\section{Desire for global harmonization of regulated bioanalysis in China}

As the pharmaceutical market has become truly global in the last decade, so have the $\mathrm{R} \& \mathrm{D}$ activities for innovative drugs. Conducting both preclinical good laboratory practice (GLP) toxicology studies and clinical trials for new chemical entities (NCEs) in China has experienced a surge of interest in recent years, primarily due to the huge potential Chinese market, along with a perceived low cost. For foreign generic drug companies, the potential large cost saving for conducting bioequivalence (BE) studies in China has also been attractive. In the meantime, Chinese pharmaceutical companies have been supplying active pharmaceutical ingredients (APIs) to other countries for many years. There is a trend for them to conduct BE studies based on international standards so the generic drugs can be approved in western countries.

Since data from the above studies might be submitted to different regulatory agencies, the bioanalytical laboratories supporting these studies in China will have to face the difficulties of dealing with many different regulations and complying with both local and international guidelines. Therefore, there is a great desire for them to follow the same harmonized bioanalytical regulations if possible.

This desire is aligned with the current efforts from the international bioanalytical community to harmonize the practice for bioanalytical method validation (BMV) and sample analysis [1-3]. Until December 2009, the US FDA 2001 guidance on BMV was the only regulatory guidance dedicated to bioanalysis in the world [4]. There have been several conferences and workshops for further clarification of this guidance published in recent years in the form of white papers, conference reports or recommendations [5-7]. Recently, the European Medicines
Agency (EMA) released its own draft guideline on $\mathrm{BMV}$ [8]. It generated a great response from many bioanalytical organizations including the EBF (European Bioanalytical Forum), the American Association of Pharmaceutical Scientists (AAPS), the Calibration Validation Group (CVG) and the Boston Society for Advanced Therapeutics (BSAT). An open letter from these organizations was published in Bioanalysis in February 2010, calling for collaboration from health authorities worldwide to work towards a global harmonization of the guidelines on BMV and sample analysis for both preclinical and clinical studies [1]. Since then, a number of meetings have been set up to discuss various aspects of the BMV guidelines with the aim of developing a harmonized guideline. At the CVG workshop in April 2010, the Global Bioanalysis Consortium (GBC) was instituted with the mission of creating one unified consensus document that could be presented to regulatory bodies/health authorities in various countries.

Bioanalytical scientists in China understand the importance of this initiative. Both the Shanghai Bioanalytical Discussion Group (SBDG) and the Beijing Bioanalytical Discussion Group (BBDG) had internal meetings to discuss this topic. The first action these two groups identified was to send a representative to join the steering committee of the GBC. To generate more awareness of bioanalytical harmonization in China, the SBDG will work with the CVG as co-organizers of the first workshop on recent issues in GLP regulated bioanalysis to be held in Shanghai in January 2011. This will be the first event on regulated bioanalysis in China, and even in the Asia-Pacific area. It is expected that this meeting will have a major impact on the Chinese State Food Drug Administration's (SFDA) regulation in bioanalysis.

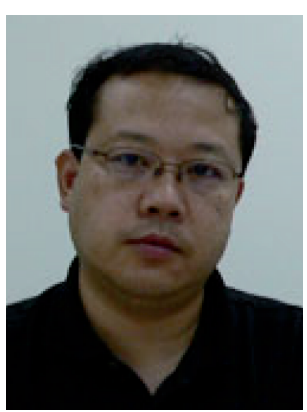

\section{Daniel Tang}

Author for correspondence: Frontage (Shanghai) Laboratories, Building 13, Lane 67 Libing Road, Shanghai 201203, China

Tel.: +862150796605 Fax: +862150796603

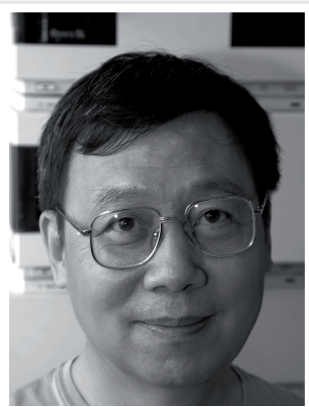

Dafang Zhong

Shanghai Institute of Materia Medica, Chinese Academy of Sciences, 50I Haike Road, Shanghai 201203, China 
To make the harmonization of bioanalytical guidelines a truly global effort, it is important that regions outside the EU and North America are equally represented in the process. Although the 2001 US FDA BMV guidance often serves as the basis of the guidelines from these regions, there are some differences among them. Therefore, it is important to understand where these existing or emerging guidelines now stand and which directions they are moving towards. The purpose of this paper is to introduce Chinese bioanalytical regulations, the current status of these GLP bioanalytical practice and future direction.

\section{Review of Chinese regulated bioanalytical guidelines}

The history of regulated bioanalysis in China spans only approximately 10 years, starting with the first GLP regulation issued by the State Drug Administration (SDA, changed to SFDA in 2003) in 1999 [9]. Before 1999, bioanalysis was mainly applied to sample analysis for clinical pharmacokinetic studies but rarely followed any international guidance. The SFDA has issued several GLP/good clinical practice (GCP) regulations, including regulation for nonclinical pharmacokinetic (PK) studies for investigational new drug (IND) submission (2005) and clinical bioavailability/bioequivalence (BA/BE) studies (2007 and 2009). As a result, the importance of following bioanalysis in a GLP environment was established and implemented rapidly in China.

Currently there are three guidelines from the SFDA that are related to bioanalysis: one was written for nonclinical research [101], whilst the other two were for clinical development [102,103], all published in 2005. In addition, the Pharmacopoeia of the People's Republic of China introduced guidelines for clinical $\mathrm{BA}$ and BE studies in 2000 with bioanalytical method validation included. They were amended twice in 2005 and 2010 [10]. None of the above guidelines were solely dedicated to bioanalysis. Instead, bioanalysis was only part of the guidelines for nonclinical PK, clinical $\mathrm{PK}$ and $\mathrm{BA} / \mathrm{BE}$ studies. It is noted that only chromatograpic methods for small molecules were discussed in detail in all guidelines. The ligand-binding assays were only mentioned very briefly in one of the guidelines [101].

The above guidelines are essentially the same and all written based on the 2001 US FDA guidance. TABLE I compares the guidelines, best practices or recommendations from China
(SFDA [101-103]), the USA (including 2001 US FDA guidance [4], 2007 white paper [5], 2009 AAPS white paper [6]) and Europe (EMA draft paper $[8])$. The focus of this article is to discuss the differences in selected areas. Only small-molecule bioanalysis is discussed as there is insufficient information from the SFDA guidelines on ligand-binding assays.

\section{- Regulatory compliance}

Like the US FDA guidelines, the SFDA guidelines do not address the issue of whether BMV is GLP compliant or not. The fact that bioanalytical method validation is discussed in both preclinical and clinical guidelines seems to imply that the agency is separating these two areas. It is noted that since September 2003, the SFDA requires that nonclinical toxicokinetic (TK) studies and clinical BA/BE studies should be conducted in compliance with GLP and GCP, respectively. For sample analysis, it is not clear in SFDA guidelines whether bioanalysis for human samples falls within the scope of GLP.

EMA draft guidelines clearly point out that both method validation and sample analysis should be conducted to GLP standards for nonclinical studies, while human bioanalytical studies fall outside of the scope of GLP and should follow GCP principles.

\section{- Scope of method validation}

The scope of the SFDA guidelines for full validation is the same as the US FDA and the EMA. For GLP TK studies in which multiple species are used, both the SFDA and the FDA guidelines state that only partial validation is required when different species are used. It is noted that the EMA requires a full validation for species changes.

\section{Reference standard \& internal standard}

Although the SFDA guidelines state that the information on Certificate of Analysis (e.g., purity, source and stability) for the test article, metabolite and internal standard should be documented in the description of the analytical method, there is no specific requirement on what kind of information must be included. The 2001 US FDA guidelines [4] specifies the quality requirement for reference standards but states no requirement for internal standard. The EMA draft guidelines move one step further - requirement for both reference and internal standards are specified. Moreover, stable-labeled internal standards are recommended whenever possible [8]. 


\section{Table 1. Comparison of method validation guidelines from the SFDA, US FDA and EMA (draft).}

\begin{tabular}{|c|c|c|c|}
\hline Topics & China SFDA (current) ${ }^{\dagger}$ & US FDA (current) ${ }^{\ddagger}$ & EMA (draft) $)^{\S}$ \\
\hline Scope (GLP) & Not defined & Not defined & $\begin{array}{l}\text { Method validation should be conducted based } \\
\text { on GLP requirement }\end{array}$ \\
\hline $\begin{array}{l}\text { Full/partial } \\
\text { validation }\end{array}$ & $\begin{array}{l}\text { Partial validation required when } \\
\text { changing species }\end{array}$ & $\begin{array}{l}\text { Partial validation required } \\
\text { when changing species }\end{array}$ & Full validation required when changing species \\
\hline RS and IS & No requirement for either RS or IS & $\begin{array}{l}\text { Requirement on quality for } \\
\text { RS but IS is not specified }\end{array}$ & $\begin{array}{l}\text { Requirement for both RS and IS is specified } \\
\text { and stable labeled IS is recommended } \\
\text { whenever possible }\end{array}$ \\
\hline Accuracy & $\begin{array}{l}\text { Demonstrated by a minimum of three } \\
\text { concentrations and minimum of five } \\
\text { replicates per concentrations }\end{array}$ & $\begin{array}{l}\text { Demonstrated by minimal } \\
\text { three concentrations and } \\
\text { minimum of five replicates } \\
\text { per concentrations }\end{array}$ & $\begin{array}{l}\text { Besides requirement of US FDA guideline, } \\
\text { recommend to demonstrate accuracy of QC } \\
\text { samples over at least one run with size } \\
\text { equivalent to a prospective analytical run }\end{array}$ \\
\hline Precision & $\begin{array}{l}\text { Demonstrated by three QC levels (LQC, } \\
\text { MQC and HQC) with at least five } \\
\text { samples per QC level }\end{array}$ & $\begin{array}{l}\text { Demonstrated by three QC } \\
\text { levels (LQC, MQC and } \\
\text { HQC) with at least five } \\
\text { samples per QC level }\end{array}$ & $\begin{array}{l}\text { Demonstrated by four QC levels (LLOQ, LQC, } \\
\text { MQC and HQC) }\end{array}$ \\
\hline $\begin{array}{l}\text { Calibration curve } \\
\text { and dynamic } \\
\text { range }\end{array}$ & $\begin{array}{l}\text { Standards prepared in the same matrix } \\
\text { as the samples } \\
\text { At least five standards in calibration } \\
\text { curve with LLOQ defined as the } \\
\text { concentration sufficient to determine } \\
\text { drug concentration at three- to } \\
\text { five-times of } T 1 / 2 \text { or } 1 / 10-1 / 20 \text { of } C_{\max } \\
\text { No requirement on the } \\
\text { regression model }\end{array}$ & $\begin{array}{l}\text { Standards prepared in the } \\
\text { same matrix as the samples } \\
\text { six to eight standards in the } \\
\text { calibration curve } \\
\text { Use the simplest regression } \\
\text { model and selection of } \\
\text { weighting. The use of more } \\
\text { complex regression needs } \\
\text { to be justified }\end{array}$ & $\begin{array}{l}\text { Freshly prepared standards are recommended } \\
\text { in validation } \\
\text { Minimum of six standards in the } \\
\text { calibration curve } \\
\text { No requirements on regression model }\end{array}$ \\
\hline Recovery & $\begin{array}{l}\text { Should be assessed at LQC, MQC and } \\
\text { HQC levels }\end{array}$ & Should be assessed & No requirement \\
\hline Dilution integrity & $\begin{array}{l}\text { No specific requirement for dilution } \\
\text { integrity in method validation }\end{array}$ & $\begin{array}{l}\text { No specific requirement } \\
\text { for dilution integrity in } \\
\text { method validation }\end{array}$ & $\begin{array}{l}\text { Should be demonstrated with acceptable } \\
\text { accuracy and precision }\end{array}$ \\
\hline Cross-validation & Not specified & $\begin{array}{l}\text { No specific procedure and } \\
\text { acceptance criteria }\end{array}$ & $\begin{array}{l}\text { Same set of QCs should be evaluated at } \\
\text { different test sites } \\
\text { Difference between test sites should be }<15 \%\end{array}$ \\
\hline
\end{tabular}




\section{Table 1. Comparison of method validation guidelines from SFDA, US FDA and EMA (draft) (cont.).}

\begin{tabular}{|c|c|c|c|}
\hline Topics & China SFDA (current) ${ }^{\dagger}$ & US FDA (current) ${ }^{\ddagger}$ & EMA (draft) $)^{\S}$ \\
\hline Matrix effects & $\begin{array}{l}\text { Need to assess but no specific } \\
\text { way to do matrix tests }\end{array}$ & $\begin{array}{l}\text { Determining MF is required for } \\
\text { Six individual lots of the matrix } \\
<15 \% \text { variation of MF is required } \\
\text { The requirement of determining } \\
\text { MF can be waived if the matrix is } \\
\text { rare or stable labeled IS is used in } \\
\text { the assay }\end{array}$ & $\begin{array}{l}\text { Determining MF is required for at least six lots of } \\
\text { matrix including haemolysed, hyperlipidaemic and if } \\
\text { applicable, from special populations } \\
\text { MF for standard and IS should be assessed } \\
\text { The concentration of maximum three-times LLOQ of } \\
\text { test compound is specified for MF assessment } \\
\text { If matrix is hard to obtain, matrix effect still need to } \\
\text { be assessed }\end{array}$ \\
\hline $\begin{array}{l}\text { Sample } \\
\text { analysis }\end{array}$ & No discussion on ISR & $\begin{array}{l}\text { ISR is required for both preclinical } \\
\text { toxicology and clinical studies in } \\
2007 \text { white paper and } 2009 \\
\text { AAPS workshop report }\end{array}$ & $\begin{array}{l}\text { Similar requirement as FDA } 2007 \text { white paper } \\
\text { Study samples close to } C_{\max } \text { and in the elimination } \\
\text { phase are recommended to be selected for ISR }\end{array}$ \\
\hline
\end{tabular}

- Selectivity, accuracy \& precision

The SFDA guidelines for selectivity, accuracy and precision are similar to FDA guidelines. It is noted that the SFDA specifically requests the inter-run results for accuracy and precision calculations should come from three consecutive runs that are prepared in three different days with no less than 45 samples. For the selectivity test, it does not discuss the possibility of back-conversion of a metabolite into parent analyte during the successive steps of analysis which is a critical point discussed in the EMA guidelines [8].

\section{- Lower limit of quantitation \& calibration curve}

Since the SFDA guidelines on bioanalysis are part of PK studies, the LLOQ in a bioanalytical assay is clearly defined as the concentration that is sufficient to determine drug concentration at three- to five-times of $T_{1 / 2}$ or $1 / 10-1 / 20$ of $\mathrm{C}_{\max }$. This assumes that the bioanalytical assay is sensitive enough to meet the requirement but it does not address the situation when the sensitivity is not enough for intended LLOQ. For PK studies of NCEs in early development stage, we have to keep in mind that often PK information is not available at the time of method development and validation. However, this should not be a problem for conducting BE studies in humans since previous studies already provide the information.

2001 US FDA guideline states that the LLOQ, which should be at least five-times the response compared with blank response, should be adapted to meet the expected drug concentrations of the study samples. It raises the question when the concentration information of NCEs is not available at the time of method validation. In the EMA draft guidelines, it simply requires
LLOQ to be the lowest amount of analyte in a sample which can be quantified reliably with an acceptable accuracy and precision.

Although the calibration curve of an assay should cover the concentrations of all study samples in the SFDA guidelines, it is not mentioned how to deal with the situation when the concentrations of study samples fall into a narrow range of the calibration curve. This is the main topic in the 2007 white paper on calibration curve and quality control (QC) ranges [5], in which it is recommended either a new narrowed standard curve be used, existing $\mathrm{QC}$ concentrations be revised or additional QC samples be added to the original curves when doing sample analysis. This topic is not mentioned in the EMA guidelines.

\section{- Carryover}

As in the 2001 US FDA guidelines, the problem of carryover is not discussed in the SFDA guidelines. As the instruments are becoming more sensitive, the impact of carryover on precision and accuracy of a chromatographic assay (especially for concentrations close to LLOQ level) becomes more serious. The 2007 white paper discusses the sources of carryover and further recommends that this problem should be addressed [5]. In this regard, the EMA paper is in line with the 2007 white paper. Both papers suggest the same way to assess the carryover and point out that the randomization of samples in the injection sequence should be avoided.

\section{Matrix effect}

Matrix effect is briefly defined and required to be assessed in SFDA guidelines. However, the specific ways to perform the experiments 
are not discussed. The comprehensive recommendations for performing the matrix-effects experiments in the 2007 White Paper and EMA draft guideline have not been updated in SFDA guidelines.

\section{Stability}

Stability tests are one of the most important areas in method validation but also are the areas that need more clarification in the SFDA guidelines. The guidelines simply state that, "based on specific situations, the stability of biological samples under room temperature, frozen conditions, freeze-thaw conditions, as well as different storage periods need to be assessed in order to determine the storage condition and storage time for these samples. In addition, attention needs to be paid to the stock solution stability and post extraction stability of the analyte."

There is a lack of information on how and when to conduct these tests in the SFDA guidelines. As a result, many inexperienced scientists or laboratories are not sure how to deal with these requirements. For example, what are the QC levels in the tests (low, mid and high QCs or only low and high QCs)? What are the acceptance criteria for these QCs? Will the stability of internal standard need to be evaluated? Should the stability evaluations be evaluated against freshly prepared standard curves? Should the storage stability of an analyte at $-70^{\circ} \mathrm{C}$ need to be re-assessed if the storage stability at $-20^{\circ} \mathrm{C}$ has been established? How long should the samples be stored in frozen condition in each cycle of a freeze-thaw stability test? Can the stock solution prepared before expiration of reference standards still be used when the reference standard expires? All of these areas have been discussed and specified in either the US FDA guidelines, white papers or the EMA draft guidelines. Many of the proposed approaches in the US FDA/EMA guidelines probably need to be considered in the SFDA guidance.

\section{- Cross-validation}

As mentioned in Smith's paper, cross-validation is probably one of the most inconsistently used terms in bioanalysis [11]. This term is not mentioned in the SFDA guidelines. Instead the term 'partial validation' is used for the method transfer between labs, change of calibration range and biological matrices, dilution of biological matrix, confirmation of method selectivity after concomitant medications. This aligns well with the the US FDA guideline's definition.
The 2001 US FDA guidelines also define the term of cross-validation as, "a comparison of validation parameters when two or more bioanalytical methods are used to generate data within the same study or across different studies." It was pointed out by Smith that cross-validation was also used loosely in the 2007 white paper to describe several validation issues when different anticoagulants or animal species are used [11], which should be the scope of partial validation in the 2001 paper.

The EMA guidelines clearly state that, "when data are obtained from different study sites, comparisons of those data are needed, and a cross-validation of the applied analytical methods should be carried out." Furthermore, it provides the specific way to do cross-validation in which the same set of QC samples should be analyzed by both analytical methods and the difference between the two measurements should not exceed $15 \%$.

Clearly the definition of cross-validation and the way to conduct cross-validation needs to be harmonized in different guidelines. Since more and more CROs or analytical labs in clinical centers are supporting multicenter studies in China, how to make sure the data obtained from different analytical labs consistent becomes a major concern.

\section{Current status of regulated bioanalysis in China}

First, the SFDA guidelines generally follow the 2001 US FDA guidelines, but they require a number of improvements. The SFDA guidelines cover most of the commonly requested evaluations. Similar to the 2001 US FDA guidelines, the SFDA guidelines do not provide specific ways to conduct many of the tests in the method validation. Some of these evaluations are only mentioned very briefly without sufficient background information, making interpretation of what is required very difficult. In the USA and Europe, the lack of detailed guidance might not be a problem because there are many bioanalytical scientists in the field who understand the issues and will follow general industrial practice (even though there are still some areas yet to reach consensus). Considering the fact that in China there are not many experienced bioanalytical scientists who are familiar with the industrial practice, the interpretation of the SFDA guidance and putting it into practice by these scientists could become a real issue. 
In addition, the SFDA guidelines do not specify acceptance criteria for many of these evaluations, including specificity, standards in standard curve, stability tests (i.e., room temperature, freezethaw, autosampler, stock solutions or long-term storage), recovery and so on. Again, this could lead to confusion among Chinese scientists.

Some of the newest developments in recent years [5-8] are yet to be reflected in the most recent SFDA or People's Republic of China Pharmacopoeia guidelines. These include areas such as matrix effects, requirement for reference standards and internal standard, new requirement for stability tests, incurred sample re-analysis and cross-validation. This confirms the importance of implementing harmonized bioanalytical guidance in China.

Second, the degree of GLP compliance in bioanalytical laboratories in China can vary dramatically. According to a recent publication, the compliance of a local Chinese bioanalytical laboratory is generally weak [9]. The situation has been improving quickly in recent years mainly due to the following reasons:

- Many 'returnees' with several years' practical experience in regulated bioanalysis in the west have returned to China, bringing back the technical know-how and regulatory understanding. These scientists are holding key positions in many CROs and pharmaceutical companies.

- Several global CROs in GLP bioanalysis have set up businesses in China, implementing the same quality systems in their Chinese laboratories as their counterparts in the USA or Europe. Many global pharmaceutical companies have chosen these laboratories for their bioanalycal work when evaluating the in-life GLP toxicology facilities in China or supporting their clinical trials.

- A few leading bioanalycal labs in research institutes or clinical centers have accumulated enough experiences by conducting GLP studies for foreign companies and learning from their colleagues from western countries in the field.

- Many training classes, or seminars, on GLP bioanalysis have been provided by CROs, instrument vendors and other nonprofit organizations such as SBDG.

As a regulatory agency, the SFDA has tightened its GLP/GCP regulations. It conducted onsite audits for all nonclinical PK studies for IND application starting from 2005. From January 2007 , it started to conduct on-site audits for all clinical BA/BE studies. From February 2009, the SFDA required all applicants to submit $100 \%$ chromatograms for BA/BE studies. All these regulations have also greatly helped improve the quality for Chinese bioanalytical practices for local laboratories.

\section{Recommendations for bioanalytical regulation harmonization}

As pointed out by Bansal et al. in a recent editorial article, it would be of great benefit to the industry and regulatory agencies if bioanalytical guidance was harmonized among major regulatory agencies [2]. The commitment from both regulatory agencies and the practitioners of bioanalysis will be the key to achieve this goal.

To start the process, it is important the awareness of harmonized bioanalytical guidelines be generated repetitively and clearly in China. The workshop organized by CVG and SBDG next January in Shanghai will surely create awareness in the SFDA and the Chinese bioanalytical community. In addition, the progress of harmonization should be reported regularly in local mass spectrometry interest groups, such as the SBDG and the BBDG, as well as several national conferences, such as the National Conference of Mass Spectrometry and the National Conference for Metabolism Studies of Drugs and Xenobiotics.

To get a broader consensus during the process of standardizing the regulation, it is also important for the involvement of scientists and regulatory agencies from other regions outside the USA and Europe. Although the USA and European scientists and regulatory agencies (US FDA and EMA) will lead the process, the input from other regions must be considered. Some of the aspects in the bioanalytical guidelines might be specifically related to the local law and government policies and should be addressed carefully.

There is a strong desire for China's bioanalytical community to have harmonized regulation. Implementation of the regulation might be lengthy, but as a Chinese proverb says, "a journey of a thousand miles begins with a single step."

Financial \& competing interests disclosure The authors have no relevant affliations or financial involvement with any organization or entity with a financial interest in or financial conflict with the subject matter or materials discussed in the manuscript. This includes employment, consultancies, honoraria, stock ownership or options, expert testimony, grants or patents received or pending, or royalties.

No writing assistance was utilized in the production of this manuscript. 


\section{Bibliography}

1 Timmerman P, Lowes S, Fast DM, Garofolo F. Request for global harmonization of the guidance for bioanalytical method validation and sample analysis. Bioanalysis 2(4), 683 (2010).

2 Bansal S, Arnold M, Garofolo F. International harmonization of bioanalytical guidance. Bioanalysis 2(4), 685-687 (2010).

3 van Amsterdam P, Lausecker B, Luedtke S, Timmerman P, Brudny-Kloeppel M. Towards harmonized regulations for bioanalysis: moving forward! Bioanalysis 2(4), 689-690 (2010).

4 US FDA. Guidance for industry: bioanalytical method validation. US Department of Health and Human Services. US FDA CDER, MD, USA (2001).

5 Viswanathan CT, Bansal S, Booth B et al. Quantitative bioanalysis methods validation and implementation: best practices for chromatographic and ligand-binding assays. AAPS J. 9(1), E30-E42 (2007).

6 Fast D, Kelley M, Viswanthan C et al. Workshop report and follow-up-AAPS workshop on current topics in GLP bioanalysis: assay reproducibility for incurred samples - implications of Crystal City recommendations. AAPS J. 11(2), 238-241 (2009).

7 Savoie N, Booth BP, Garofolo F et al. The 2nd calibration and validation group workshop on recent issues in good laboratory practice bioanalysis. Bioanalysis 1(1), 19-30 (2009).

8 European Medicine Agency. Draft Guideline on Validation of Bioanalycal Methods. EMEA/CHMP/EWP/192217/2009 (2009).

9 Zhong DF. Bioanalysis in China. Bioanalysis, 2(2), 153-156 (2010).

10 Chinese Pharmacopoeia Commission. Pharmacopoeia of the People's Republic of China (2010) Vol. II, Appendix XIX B. China Medical Science and Technology Press, Beijing, China (2010).

11 Smith G. Bioanalytical method validation: notable points in the 2009 draft EMA guideline and differences with the 2001 US FDA Guidance. Bioanalysis 2(5), 929-935 (2010).

\section{- Websites}

101 China SFDA. Technical guideline on non-clinical pharmacokinetic studies for chemical drugs (2005). www.sda.gov.cn/gsz05106/15.pdf

102 China SFDA. Guideline on human bioavailability and bioequivalence studies for chemical drug formulation (2005). www.sda.gov.cn/gsz05106/08.pdf

103 China SFDA. Technical guideline on clinical pharmacokinetic studies for chemical drugs (2005). www.sda.gov.cn/gsz05106/07.pdf

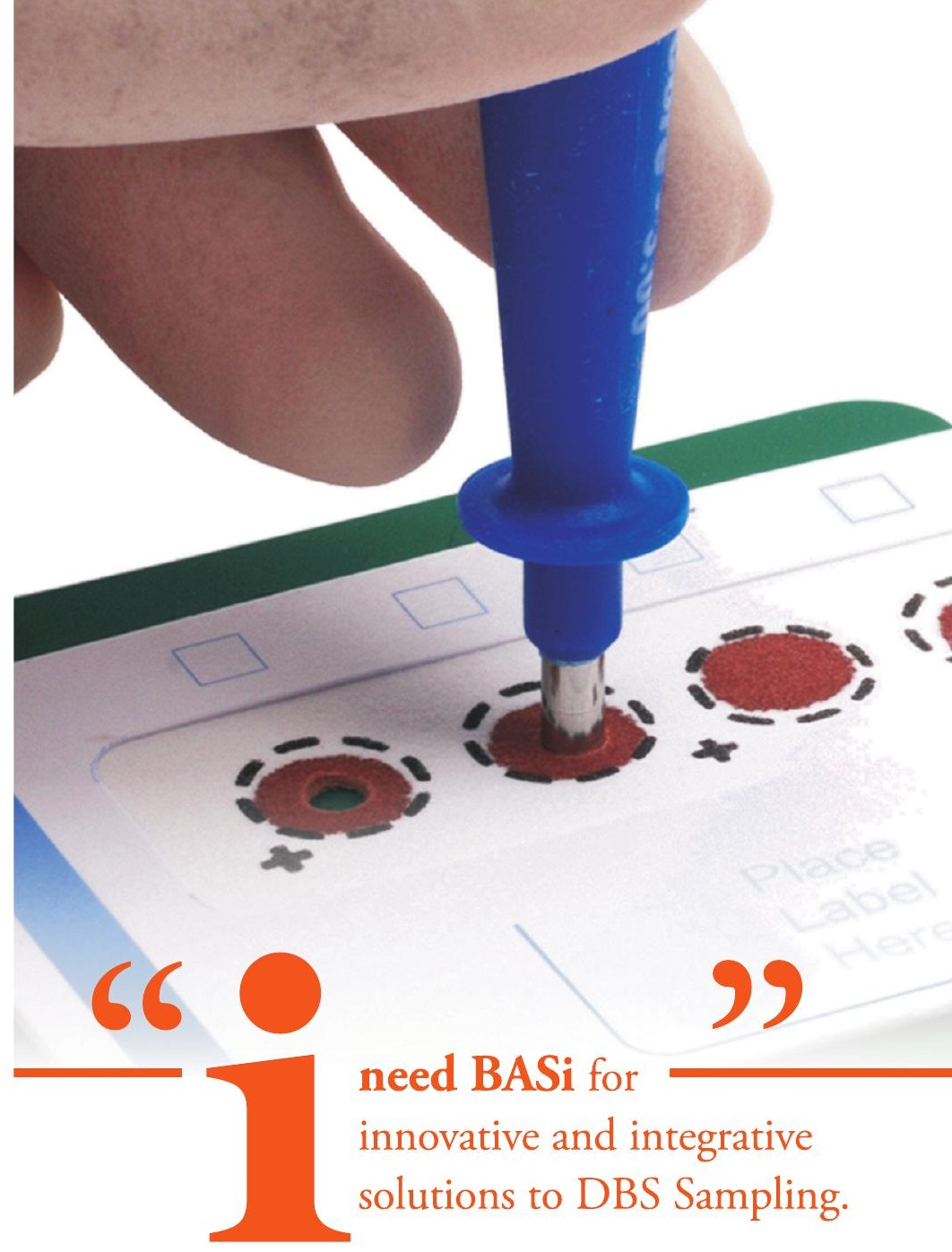

- Small volume collection, storage, shipping of blood samples

- Reduction of experimental animals

- Low stress sampling for mice to men

UNITED KINGDOM

Suite 2, Building 500

Abbey Park, Stareton, Kenilworth

Warwickshire, UK CV8 2LY

+44 (0) 2476639574

CORPORATE CENTER

2701 Kent Avenue

West Lafayette, IN 47906

$800.845 .4246 \mid 1+765.463 .4527$

basi@BASInc.com

www.BASInc.com 